\title{
Massive Chylous Ascites and Chylothorax Secondary to Chronic Pancreatitis: A Novel Surgical Option
}

\author{
Keith D. Lillemoe, MD, FACS ${ }^{1}$ \\ ${ }^{1}$ Division of Surgical Oncology, Department of Surgery, \\ Massachusetts General Hospital, Boston, Massachusetts \\ 2 Division of Plastic Surgery, Department of Surgery, Massachusetts \\ General Hospital, Boston, Massachusetts \\ ${ }^{3}$ Division of Thoracic Surgery, Department of Surgery, Massachusetts \\ General Hospital, Boston, Massachusetts \\ ${ }^{4}$ Division of Interventional Radiology, Department of Radiology, \\ Massachusetts General Hospital, Boston, Massachusetts
}

Grace C. Lee, MD ${ }^{1}$ Curtis L. Cetrulo, Jr., MD, FACS, FAAP ${ }^{2}$ Ashok Muniappan, MD ${ }^{3}$ Kei Yamada, MD

Address for correspondence Keith D. Lillemoe, MD, Department of Surgery, Massachusetts General Hospital, 15 Parkman Street, Boston, MA 02114 (e-mail: klillemoe@mgh.harvard.edu).

J Reconstr Microsurg Open 2020;5:e22-e26.

\begin{abstract}
Keywords

- chylous ascites

- chyle leak

- lymphatic flap

Chylous ascites is a debilitating condition characterized by milky, triglyceride-rich fluid accumulating in the peritoneal cavity due to disruption of the thoracic or intestinal lymphatic system. ${ }^{1}$ It is associated with severe malnutrition, immunosuppression, and mortality, ${ }^{2}$ and unfortunately one-third of patients do not respond to medical therapies. ${ }^{3}$ We present a rare case of massive chylous ascites and chylothorax secondary to chronic pancreatitis in a patient who failed multiple medical therapies, and who was ultimately successfully treated with a novel surgical lymphatic cable flap.
\end{abstract}

Background Chylous ascites is a debilitating condition characterized by milky, triglyceride-rich fluid accumulating in the peritoneum due to disruption of the intraabdominal lymphatic system. Medical management includes low-fat diets, somatostatin analogues, and therapeutic paracentesis, but is unsuccessful in one-third of patients.

Methods We present a 59-year-old man with massive chylous ascites and chylothorax secondary to chronic pancreatitis, who failed medical therapies for nearly two years, before being successfully treated with a novel surgical technique. Demographic and clinical data were obtained from the electronic medical record at Massachusetts General Hospital from 2015 to 2019. Patient information was kept anonymous and informed consent was obtained for publication of this report.

Results Based on a previously published small case series, we created a vascularized lymphatic cable flap based on the superior epigastric vessels, which we anastomosed to mesenteric vessels, permitting chylous drainage superiorly through the thoracic duct. With two years of follow-up, our patient has no evidence of recurrent ascites or chylothorax, and robust nutritional and functional status.

Conclusion We present this novel surgical technique as a promising intervention for patients with chylous ascites and/or chylothorax who have failed medical management. received

November 14, 2019

accepted

March 20, 2020
DOI https://doi.org/

$10.1055 / \mathrm{s}-0040-1710344$. ISSN 2377-0813.

\section{Case Report}

A 59-year-old man with history of hypertension and prostate cancer status postremote radical prostatectomy was referred to the Massachusetts General Hospital (MGH) for management of chylous ascites and chylothorax secondary to severe pancreatitis that likely damaged the cisterna chyli.

The patient had severe gallstone pancreatitis in September 2014, which required an intensive care unit stay at an outside hospital, during which time he underwent a laparoscopic

Copyright @ 2020 by Thieme Medical Publishers, Inc., 333 Seventh Avenue, New York, NY 10001, USA. Tel: +1(212) 760-0888.
License terms

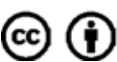



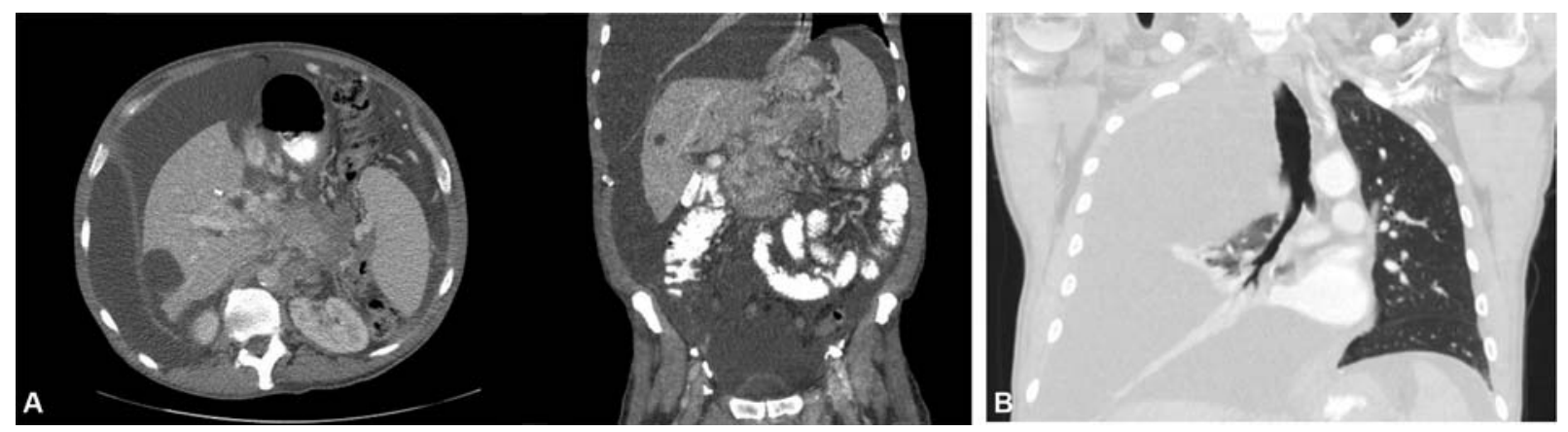

Fig. 1 Computed tomography images of the (A) abdomen and (B) chest obtained prior to surgical intervention, demonstrating severe ascites, right chylothorax, and right lung collapse.

cholecystectomy. He recovered well, but in April 2015, he developed dyspnea and was found to have a large right-sided pleural effusion. He underwent thoracentesis, with removal of $3 \mathrm{~L}$ of chyle. He had no abdominal pain; however, axial imaging demonstrated extensive pancreatic necrosis consistent with his prior pancreatitis; portal vein, superior mesenteric vein, and splenic vein thrombosis; extensive ascites; a possible defect in the right hemidiaphragm; and a large right pleural effusion with associated collapse of the right lower lobe ( - Fig. 1). There was no pancreatic ductal dilatation to suggest malignancy or obstruction.

Attempts were first made to drain the chyle that had already collected in his peritoneum and pleural cavity, and slow the production of chyle. He was admitted to the MGH in September 2015, where he underwent percutaneous rightsided chest tube placement and was started on a nonfat clear liquid diet, fat-free total parenteral nutrition (TPN), and octreotide. Triglyceride levels in the pleural fluid decreased from 244 to $17 \mathrm{mg} / \mathrm{dL}$, and cytology demonstrated no malignant cells. There was almost complete resolution of his ascites. His chest tube was removed on the day of discharge. However, though he continued a low-sodium, nonfat diet supplemented with medium-chain triglycerides after discharge, the chylothorax and chylous ascites recurred.

In October 2015, he underwent a lymphangiogram, which opacified the cisterna chyli and demonstrated no evidence of a leak. Several transabdominal attempts were made to access the cisterna chyli, in addition to attempts to cannulate the thoracic duct, without success. He was admitted in December 2015 and again underwent chest tube placement, and was started on a nonfat diet, TPN, and octreotide. Again, there was temporary improvement, but the ascites and chylothorax recurred soon after discharge.

The hepatology service suggested that his ascites and effusion may be due in part to cirrhosis and portal hypertension. Transjugular liver biopsy demonstrated no cirrhosis. In March 2016, he underwent portal vein recanalization and stent placement. The accumulation of ascites slowed but continued, and he required paracenteses and thoracenteses with increasing frequency. Repeat axial imaging in August 2016 demonstrated partial in-stent thrombosis. During this time, he developed increasingly large and uncomfortable bilateral inguinal hernias and an umbilical hernia, as well as worsening dyspnea due to repeated reaccumulation of the chylothorax. These symptoms were particularly debilitating given that the patient was recently widowed and the sole provider for two young twin boys. His nutritional and functional status began deteriorating, and his overall health was reaching a point of chronic critical illness, with his serum albumin declining to $2.5 \mathrm{~g} / \mathrm{dL}$.

At this point, the decision was made to attempt repair of the inguinal and umbilical hernias along with a novel lymphatic cable flap to prevent reaccumulation of chylous ascites. This flap, proposed by Chen et al, is designed to divert lymph away from the leak by transferring the superior epigastric vessels with surrounding lymphatic fatty tissue into the abdominal cavity and anastomosing them to mesenteric vessels, thereby permitting chyle to drain superiorly through the lymph node chain of the flap and into the thoracic duct (-Fig. 2). ${ }^{4}$

The patient was admitted in January 2017, 1 week prior to scheduled surgery. He underwent paracentesis with removal of $8.4 \mathrm{~L}$ of chylous ascites, along with right chest tube placement. He was started on a nonfat clear liquid diet, octreotide, and TPN to improve his nutritional status prior to surgery. He was taken to the operating room and undergone exploratory laparotomy via a small midline incision, with removal of $6 \mathrm{~L}$ of ascites. No chyle leak was identified, even with a heavy cream challenge the night before and via orogastric tube in the operating room. Further exploration of the retroperitoneum was deferred due to the significant inflammatory mass surrounding the pancreas and extensive venous collaterals from portal venous thrombosis. There was no evidence of a diaphragmatic defect.

The plastic surgery team performed the lymphatic cable flap. They dissected the right deep inferior epigastric artery and vein free from the posterior rectus muscle after lateral exposure of the muscle. Next, they raised the vascularized lymph node flap, which consisted of the right deep inferior epigastric artery and vein pedicle and its surrounding fat and lymphatic tissue. The deep inferior epigastric vessels were ligated distally just before they enter the iliac vessels to obtain length for mobility of the flap. The flap, which was technically now based on the superior epigastric vessels as its pedicle (which technically also included the choke vessels connecting the superior epigastric vessels and deep inferior epigastric vessels), was then swung medially and cephalad and placed into the abdomen via an opening in the posterior rectus sheath. The recipient vessels in the mesentery of the 

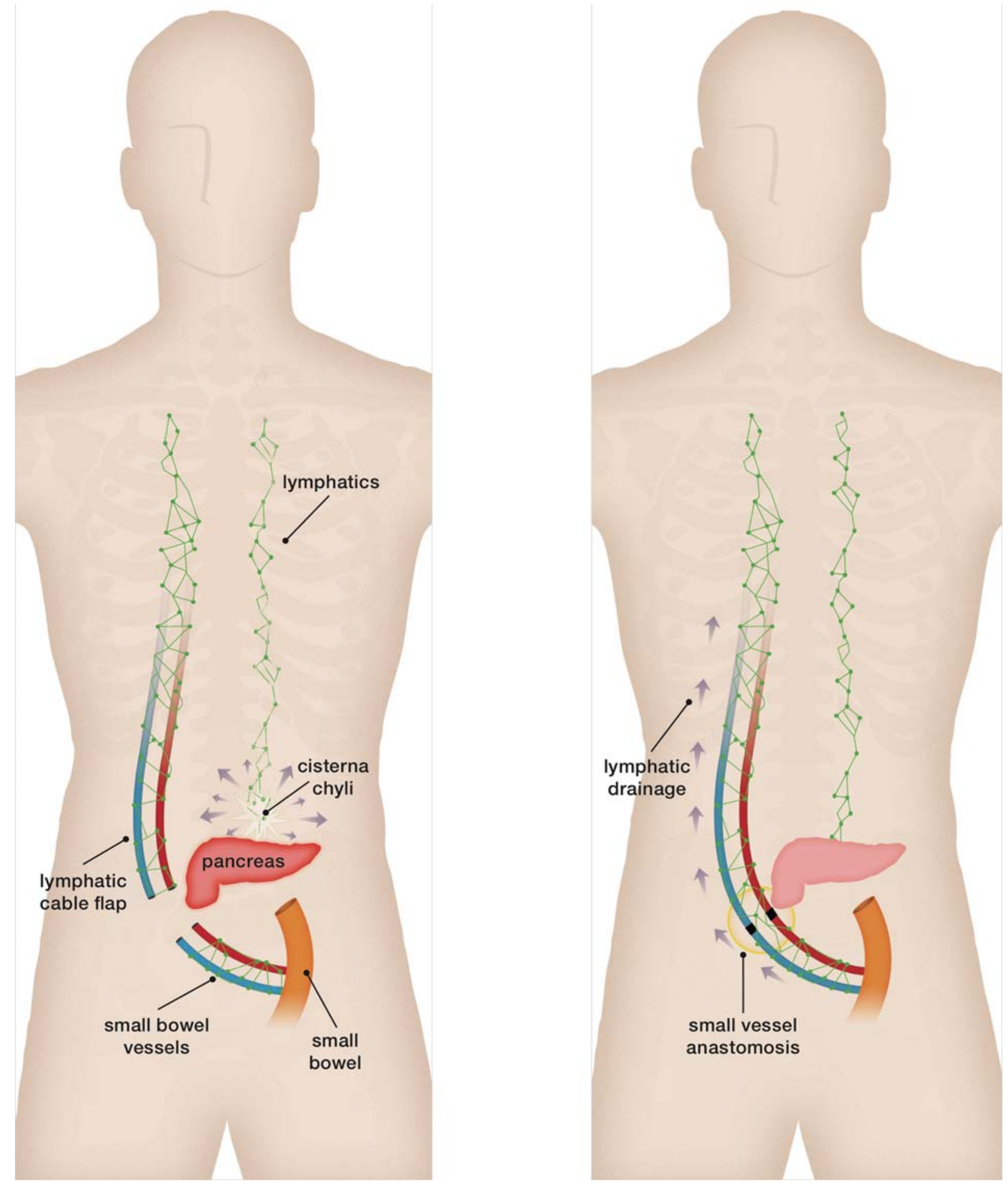

Fig. 2 Diagram depicting the lymphatic cable flap, which diverts lymph away from the leak by transferring the superior epigastric vessels (including the choke vessels connecting the superior epigastric vessels and deep inferior epigastric vessels) with surrounding fat and lymphatic tissue into the abdominal cavity and anastomosing them to mesenteric vessels, thereby permitting chyle to drain superiorly through the lymph node chain of the flap and into the thoracic duct.

ileum were prepared for microsurgical anastomosis approximately $10 \mathrm{~cm}$ proximal to the ileocolic junction. They then performed microsurgical anastomoses of the artery and vein in standard fashion, and confirmed patency with Doppler signals. At this point, the right inguinal hernia was repaired with a mesh plug through the right groin incision made for the lymphatic flap, and the left inguinal hernia was repaired with a mesh plug through a new left groin incision. Three peritoneal drains were placed, and the umbilical hernia was repaired primarily with closure of the midline incision.

The patient tolerated the procedure well, though required aggressive fluid resuscitation. He recovered in the intensive care unit and quickly weaned off pressor support. He was advanced to a low-fat diet, and received octreotide and TPN 

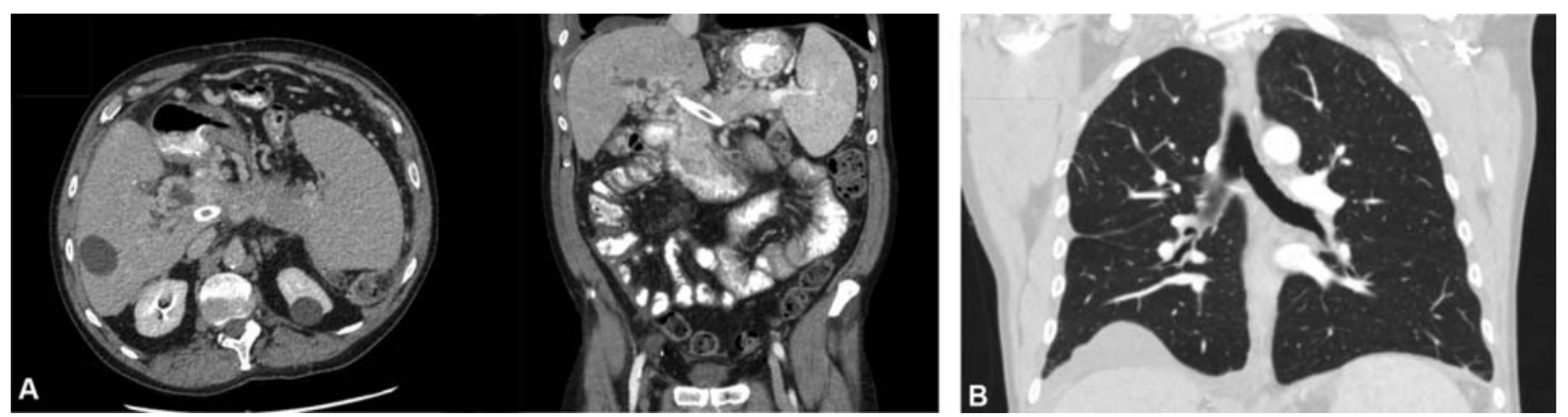

Fig. 3 Computed tomography images of the (A) abdomen and (B) chest obtained 2 years after surgical intervention, demonstrating resolution of ascites and chylothorax and expansion of right lung.

until the day of discharge. He initially had high serous peritoneal drain output, which decreased with diuretic therapy. All peritoneal drains and the chest tube were removed prior to discharge on postoperative day 10 . He was started on aspirin $81 \mathrm{mg}$ daily because of the small vessel anastomoses of the lymphatic cable flap.

One month later the patient was doing well, with no evidence of wound drainage, surgical site infection, hernia recurrence, or ascites or chylothorax reaccumulation on radiographs. His nutritional status improved, with serum albumin increasing to $3.1 \mathrm{~g} / \mathrm{dL} 1$ year postoperatively. With over 2 years of follow-up, the patient has continued to do well, with near complete resolution of ascites and chylothorax, and significant reexpansion of his right lung (-Fig. 3). He has not required any further paracentesis or thoracentesis. He has no dietary restrictions but does continue to take diuretics. He has been very active, working full-time, and caring for his twin boys.

\section{Discussion}

Chylous ascites is the accumulation of triglyceride-rich fluid in the peritoneum due to the disruption of the thoracic or intestinal lymphatic system. ${ }^{1}$ The causes of this lymphatic disruption are diverse but can be categorized into three principal mechanisms: (1) obstruction of lymphatics due to fibrotic or infiltrative processes such as malignancy, leading to exudation of chyle through the walls of dilated lymphatic channels; (2) lymphatic disruption due to trauma or surgery, causing direct leakage of chyle through a lymphoperitoneal fistula; and (3) exudation of chyle through dilated retroperitoneal lymphatics due to congenital lymphangiectasia or thoracic duct obstruction. ${ }^{2}$ In developing countries, the main causes of chylous ascites are infections such as tuberculosis, while in developed countries the primary causes are malignancy, cirrhosis, and trauma. ${ }^{5}$ The incidence of chylous ascites has been increasing, ${ }^{6}$ likely due in part to the performance of increasingly aggressive thoracic, abdominal, and retroperitoneal operations. ${ }^{2,7}$

Pancreatitis is a rare cause of chylous ascites, with less than ten cases reported in the literature. ${ }^{8}$ Chylous ascites likely results from either disruption of retroperitoneal lymphatics by pancreatic enzymes or from obstruction of lymphatics due to massive peripancreatic inflammation and eventual fibrosis. When it occurs, chyloperitoneum tends to appear days to weeks after the diagnosis of pancreatitis, ${ }^{8}$ as with our patient.
Chylous ascites is a debilitating condition associated with mortality rates as high as 43 to $71 \%$, though rates have been reported to be lower in cases of postoperative chyle leaks. ${ }^{2}$ The leakage of chyle prevents the return of protein to the systemic circulation, leading to severe protein-losing enteropathy and malnutrition. Patients also often become immunocompromised due to loss of lymphocyte-rich fluid, in addition to developing hypogammaglobulinemia from protein losses, thus increasing their susceptibility to infections. ${ }^{2}$ Respiratory embarrassment may occur from diaphragmatic dysfunction as ascites builds and also from chyle accumulation in the pleural spaces, presumably from natural diaphragmatic channels that may exist.

The management of chylous ascites begins with accurate diagnosis, treatment of the underlying cause, and preservation of the patient's nutritional status. Diagnosis of chylous ascites is variably reported as an ascitic triglyceride concentration of $>110$ or $>200 \mathrm{mg} / \mathrm{dL}^{1,4}$ Medical management of chylous ascites initially comprises dietary adjustments to reduce the production of chyle. Patients are placed on a low-fat, high-protein diet with supplementation of medium-chain triglycerides (which are absorbed directly into portal venous circulation, bypassing lymphatics). ${ }^{9,10}$

In addition to dietary modifications, many patients are started on octreotide or other somatostatin analogs, which are believed to decrease portal pressure and reduce intestinal fat absorption. ${ }^{11,12}$ Other therapies have been suggested, such as glue embolization, surgical ligation, and embolization of disrupted lymphatic channels; however, data are limited and results are mixed. ${ }^{2,3,5,13}$ Surgical identification and ligation of the source of chyle leak is often unsuccessful due to extensive inflammatory or fibrotic changes and because patients often have multiple chyloperitoneal fistulas. ${ }^{4}$ Peritoneovenous shunting has been used in the past but is no longer suggested due to high rates of complications including sepsis, disseminated intravascular coagulation, and early occlusion due to the high viscosity of chyle. ${ }^{4,14}$ Unfortunately, one-third of patients with chylous ascites do not respond to medical therapies. ${ }^{3}$ Palliation is achieved by intermittent thoracentesis and paracentesis. Surgical or chemical pleurodesis may be attempted but may be ineffective or result in loculated effusions in the setting of massive chyle production, as in our patient.

In this setting, Chen et al harnessed their experience with using vascularized lymph node flaps for extremity 
lymphedema, and created a vascularized lymphatic cable flap based on the superior epigastric vessels and their surrounding fatty, lymphatic-rich tissue. ${ }^{4}$ This flap addresses the underlying lymphatic obstruction by creating an extraperitoneal bypass of the damaged lymphatics, enabling lymph and chylous ascites to drain via the superior epigastric vein and surrounding lymphatics in the thoracic wall into the thoracic duct and subclavian vein. Their operative technique consists of identifying the deep inferior epigastric vessels on the posterior aspect of the rectus abdominis muscle, and dissecting the vascular bundle with its surrounding fat and lymphatic tissue down to the vessels' origin at the external iliac. They next enter the peritoneum, isolate the fourth jejunal artery and vein, and perform end-to-end anastomoses between the deep inferior epigastric vessels and the fourth jejunal vessels. They performed this procedure in three patients with chylous ascites, with resolution of ascites, thoracic reexpansion, normalized serum albumin, and no perioperative complications. ${ }^{4}$

Our patient is an additional compelling example of the success of a lymphatic cable flap for cases of intractable chylous ascites. This technique proposed by Chen et al may be a promising surgical option to offer to patients suffering from this debilitating condition. ${ }^{4}$

\section{Funding}

G.C.L. received financial support from an NIH T32 grant (Research Training in Alimentary Tract Surgery, DK007754-13).

\section{Conflict of Interest \\ None declared.}

\section{References}

1 Lizaola B, Bonder A, Trivedi HD, Tapper EB, Cardenas A. Review article: the diagnostic approach and current management of chylous ascites. Aliment Pharmacol Ther 2017;46(09):816-824
2 Aalami OO, Allen DB, Organ CH Jr. Chylous ascites: a collective review. Surgery 2000;128(05):761-778

3 Almakdisi T, Massoud S, Makdisi G. Lymphomas and chylous ascites: review of the literature. Oncologist 2005;10(08): 632-635

4 Chen SH, Yeh LF, Ciudad P, Chen HC. Successful surgical treatment of intractable chylous ascites using the lymphatic cable flap: a retrospective review study. World J Surg 2017;41(12): 3100-3104

5 Cárdenas A, Chopra S. Chylous ascites. Am J Gastroenterol 2002; 97(08):1896-1900

6 Press OW, Press NO, Kaufman SD. Evaluation and management of chylous ascites. Ann Intern Med 1982;96(03):358-364

7 Besselink MG, van Rijssen LB, Bassi C, et al; International Study Group on Pancreatic Surgery. Definition and classification of chyle leak after pancreatic operation: A consensus statement by the international study group on pancreatic surgery. Surgery 2017 ; 161(02):365-372

8 D'Amata G, Rega M, Viola V, Bove V, Simeone P, Baiano G. Chyloperitoneum associated with idiopathic pancreatitis: case report and review of the literature. G Chir 2016;37(04): $167-170$

9 Leibovitch I, Mor Y, Golomb J, Ramon J. The diagnosis and management of postoperative chylous ascites. J Urol 2002;167 (2 Pt 1):449-457

10 Weinstein LD, Scanlon GT, Hersh T. Chylous ascites. Management with medium-chain triglycerides and exacerbation by lymphangiography. Am J Dig Dis 1969;14(07):500-509

11 Ilhan E, Demir U, Alemdar A, Ureyen O, Eryavuz Y, Mihmanli M. Management of high-output chylous ascites after D2lymphadenectomy in patients with gastric cancer: a multi-center study. J Gastrointest Oncol 2016;7(03):420-425

12 Leong RW, House AK, Jeffrey GP. Chylous ascites caused by portal vein thrombosis treated with octreotide. J Gastroenterol Hepatol 2003;18(10):1211-1213

13 Boccardo F, Bellini C, Eretta C, et al. The lymphatics in the pathophysiology of thoracic and abdominal surgical pathology: immunological consequences and the unexpected role of microsurgery. Microsurgery 2007;27(04):339-345

14 Yarmohammadi H, Brody LA, Erinjeri JP, et al. Therapeutic application of percutaneous peritoneovenous (Denver) shunt in treating chylous ascites in cancer patients. J Vasc Interv Radiol 2016;27 (05):665-673 